\title{
POWER FLOW IN DISTRIBUTION NETWORKS WITH EARTH RETURN
}

\author{
Rade M. Ciric, Luis F. Ochoa, Antonio Padilha \\ Universidade Estadual Paulista - Campus de Ilha Solteira \\ Av. Brasil 56 CEP 15385-000 Ilha Solteira, SP, Brazil
}

\begin{abstract}
The problems of wave propagation and power flow in the distribution network composed of an overhead wire parallel to the surface of the ground have not been satisfactorily solved. While a complete solution of the actual problem is impossible, as it is explained in the famous Carson's paper (1926), the solution of the problem, where the actual earth is replaced by a plane homogenous semi-infinite solid, is of considerable interest. In this paper, a power flow algorithm in distribution networks with earth return, based on backwardforward technique, is discussed. In this novel use of the technique, the ground is explicitly represented. In additon, an iterative method for determining impedance for modelling ground effect in the extended power flow algorithm is suggested. Results obtained from single-wire and three-wire studies using IEEE test networks are presented and discussed.
\end{abstract}

Keywords: Distribution networks, Single-wire with earth return, Three-wire system with earth return, Power flow

\section{INTRODUCTION}

The distribution network with earth return (DNER) presents an old-fashion technical solution with serious problems in network operation, like daily time varying ground resistivity and unexpected over voltages on the consumer's side. However, the utilities in Brazil, Australia, South Africa, Iceland, etc. still operate single wire earth return (SWER) distribution networks in the rural electrification. The reason these networks still operate is very simple: these network solutions are less expensive in compare to the standard networks, and on the 
other side the remote farms usually supplied by the SWER, can get better power quality using simple and cheap facilities, like voltage corrector.

The problems of wave propagation and power flow in the distribution network composed of one or three overhead wires parallel to the surface of the ground have not been satisfactorily solved. While a complete solution of the actual problem is impossible [1], the solution of the problem, where a plane homogenous semi-infinite solid replaces the actual earth, is of considerable interest [3], [4], [5].

In this paper, a power flow algorithm for the single-wire and three-wire DNER, based on backward-forward technique, is discussed. The main objective of this work is to demonstrate that $2 \times 2$ and $4 \times 4$ line models can be included in the backward-forward procedure [7] with minor changes. In addition, an iterative method for determining impedances of the DNER needed in developed power flow algorithm, based on simple measurement and exhaustive power flow calculation, is suggested. Results obtained from single-wire and threewire studies using IEEE test feeder, are presented and discussed.

This paper consists of seven parts. In the second part Carson's line and definition of the problem are presented for computing the ground self and mutual impedance. In the third part, the proposed power flow algorithm in the DNER, based on backward-forward technique, is described. The fourth part contains application aspects of the proposed methodology. The conclusion is in the fifth part and the final part contains the list of references. In the Appendix is suggested a method for determining impedance for modelling ground effect in the power flow algorithm.

\section{CARSON's Line}

The Carson's paper [1] describes the impedances of an overhead conductor with earth return. This famous paper was reprinted in the Proceedings of the Thirty-Second Annual North American Power Symposium [2], and the Carson's equations have become widely used in distribution system analysis [3], [4]. 
In this section, the main focus is to compute the ground self and mutual impedance of a DNER. Carson considers a single conductor $a$ one unit long and parallel to the ground (Fig. 1), carrying a current $I_{a}$ with a return through circuit $g-g^{\prime}$ beneath the surface of the earth (considered to have a uniform resistivity and to be of infinite extent). Carson's line can be thought of as single return conductor with a self geometric mean radius (GMR) of 1 foot (or 1 meter), located at a distance $D_{a g}$ feet (or meters) below the overhead line, where $D_{a g}$ is a function of the earth resistivity $\rho$ [4].

FIGURE 1.

Thus, for Carson's line:

$$
\left[\begin{array}{c}
V_{a a^{\prime}} \\
V_{g g^{\prime}}
\end{array}\right]=\left[\begin{array}{c}
V_{a}-V_{a^{\prime}} \\
V_{g}-V_{g^{\prime}}
\end{array}\right]=\left[\begin{array}{cc}
\bar{Z}_{a a} & \bar{Z}_{a g} \\
\bar{Z}_{a g} & \bar{Z}_{g g}
\end{array}\right]\left[\begin{array}{c}
I_{a} \\
-I_{a}
\end{array}\right] \mathrm{V} / \text { unit length, }
$$

where $V_{a}, V_{a^{\prime}}, V_{g}$ and $V_{g^{\prime}}$ are all measured with respect to the same reference, and $V_{g}=0$.

Then, by subtracting the two equations:

$$
V_{a}=\left(\bar{z}_{a a}+\bar{z}_{g g}-2 \bar{z}_{a g}\right) I_{a}=Z_{a a} I_{a}
$$

Hence,

$$
z_{a a} \cong \bar{z}_{a a}+\bar{z}_{g g}-2 \bar{z}_{a g}
$$

Where $\bar{Z}_{a a}$ represents the self-impedance of the line, and $\left(\bar{z}_{g g}-2 \bar{Z}_{a g}\right)$ is the correction caused by the ground presence.

Using the correction part of the simplified Carson's equations for self impedances, we obtain the following equation:

$$
\begin{aligned}
\bar{z}_{g g}-2 \bar{z}_{a g}= & \pi^{2} \cdot 10^{-4} f-j 0.0386 \cdot 8 \pi \cdot 10^{-4} f \\
& +j 4 \pi \cdot 10^{-4} \cdot f \ln \frac{2}{5.6198 \cdot 10^{-3}}+j 4 \pi \cdot 10^{-4} \cdot \ln \frac{\sqrt{\rho / f}}{h_{a}},
\end{aligned}
$$

where

$f=$ frequency;

$h_{a}=$ height of wire in meters; 
$\rho=$ ground resistivity.

Looking at the right side of Eq. (4), we can identify the terms that are just frequency dependent. These three terms represent the ground self-impedance $\bar{Z}_{g g}$. Therefore, the ground self impedance $\bar{Z}_{g g}$ and the mutual impedance $\bar{Z}_{a g}$ from Fig. 1 and Eq. (3) are

$$
\begin{aligned}
& \bar{z}_{g g}=\pi^{2} \cdot 10^{-4} f-j 0.0386 \cdot 8 \pi \cdot 10^{-4} f+j 4 \pi \cdot 10^{-4} \cdot f \ln \frac{2}{5.6198 \cdot 10^{-3}} \\
& \bar{z}_{a g}=-\frac{1}{2} \cdot j 4 \pi \cdot 10^{-4} \cdot \ln \frac{\sqrt{\rho / f}}{h_{a}}=j 2 \pi \cdot 10^{-4} \cdot \ln \frac{h_{a}}{\sqrt{\rho / f}} .
\end{aligned}
$$

In a three-phase line with ground return, the following $4 \times 4$ matrix representation of line impedance is introduced:

$$
\left[Z_{\text {line }}\right]=\left[\begin{array}{llll}
\bar{Z}_{a a} & \bar{Z}_{a b} & \bar{Z}_{a c} & \bar{Z}_{a g} \\
\bar{Z}_{a b} & \bar{Z}_{b b} & \bar{Z}_{b c} & \bar{Z}_{b g} \\
\bar{Z}_{a c} & \bar{Z}_{b c} & \bar{Z}_{c c} & \bar{Z}_{c g} \\
\bar{Z}_{a g} & \bar{Z}_{b g} & \bar{Z}_{c g} & \bar{Z}_{g g}
\end{array}\right]
$$

The elements of the $3 \times 3$ sub matrix in Eq. (7), self and mutual impedances of phases $a$, $b$ and $c$, are calculated using the Carson's equations considering the ground as a perfect conductor. E.g.: for phase $a$, the self impedance is

$$
\bar{z}_{a a}=r_{a}+j 4 \pi \cdot 10^{-4} f \ln \left(\frac{2 h_{a}}{G M R_{a}}\right) \Omega / \mathrm{km},
$$

And the mutual impedance between phases $a$ and $b$ is

$$
\bar{z}_{a b}=j 4 \pi \cdot 10^{-4} f \ln \left(\frac{\sqrt{d_{a b}^{2}+\left(h_{a}+h_{b}\right)^{2}}}{\sqrt{d_{a b}^{2}+\left(h_{a}-h_{b}\right)^{2}}}\right) \Omega / \mathrm{km}
$$

where
$r_{a} \quad$ is the resistance of phase wire $a$ in $\Omega / \mathrm{km}$;
$h_{a}, h_{b} \quad$ are the heights of phase wires $a$ and $b$ in meters;
$d_{a b} \quad$ is the horizontal distance between phase wires $a$ and $b$ in meters; 
GMRa is the geometric mean radius of phase wire $a$ in meters.

\section{Power Flow Algorithm}

Efficient and robust three-phase power flow algorithm for large-scale distribution networks with generators and loops is the basic tool for distribution management system today [5]. In last decade, different procedures for distribution power flow have been proposed [3], [6], [7], [8], [9] and [10]. Experience shows that very good results in handling large-scale distribution networks are obtained using the backward-forward procedure, i.e. branch-oriented methods. These methods may be classified as follows: current summation methods, power summation methods and admittance summation methods. In this work, the power flow method proposed in [7] is extended to model the ground explicitly in a $4 \times 4$ network representation.

\section{Model of the Distribution Network with Earth Return}

Model of the single-wire earth return and three-wire with earth return distribution networks, are presented on Fig. 2 and Fig. 3, respectively.

\section{FIGURE 2.}

FIGURE 3.

All variables on the Fig. 2 and Fig. 3 are complex and have the following meaning:

$\bar{Z}_{a a}, \bar{Z}_{b b}, \bar{Z}_{c c}$ are the series impedances of phase lines;

$\overline{\mathrm{Z}}_{g g} \quad$ is the impedance of a fictitious ground wire;

$Y_{a}, Y_{b}, Y_{c} \quad$ are the shunt admittances of phase lines;

Zload $\quad$ load impedance (in case of single-wire DNER, Fig. 2).

\section{Power Flow Algorithm in Distribution Networks with Earth Return}

The efficient and robust three-phase branch-oriented backward-forward procedure from [7] was used for power flow computation in the three wire DNER. However, $3 \times 3$ network 
representation from [7] is expanded on $4 \times 4$ network representation, considering three-phase wires and fictitious ground wire. Branch numbering scheme for radial network is exactly like in [7]. The power flow computation in the three-wire DNERs is in the following:

In iteration $k$ :

1. Nodal Current Calculation

$$
\left[\begin{array}{c}
I_{i a} \\
I_{i b} \\
I_{i c} \\
I_{i g}
\end{array}\right]^{(k)}=\left[\begin{array}{c}
\left(S_{i a} / V_{i a}^{(k-1)}\right)^{*} \\
\left(S_{i b} / V_{i b}^{(k-1)}\right)^{*} \\
\left(S_{i c} / V_{i c}^{(k-1)}\right)^{*} \\
-\left(I_{i a}^{(k)}+I_{i b}^{(k)}+I_{i c}^{(k)}\right)
\end{array}\right]-\left[\begin{array}{llll}
Y_{i a} & & & \\
& Y_{i b} & & \\
& & Y_{i c} & \\
& & & 0
\end{array}\right]\left[\begin{array}{c}
V_{i a} \\
V_{i b} \\
V_{i c} \\
V_{i g}
\end{array}\right]^{(k-1)}
$$

where

Iia, $I_{i b}, I_{i c}, I_{i g}$

$S_{i a}, S_{i b}, S_{i c}$

$V_{i a}, V_{i b}, V_{i c}, V_{i g}$

$Y_{i a}, Y_{i b}, Y_{i c}$

$k$ are the current injections at node $i$;

are the scheduled (known) power injections in phase $a, b, c$ at node $i$;

are the phase voltages and ground voltage at node $i$;

are the admittance of all shunt elements at node $i$;

is the iteration index.

2. Backward Sweep - section current calculation

Starting from the line section in the last layer and moving towards the root node, the current in line section $l$ is:

$$
\left[\begin{array}{l}
J_{l a} \\
J_{l b} \\
J_{l c} \\
J_{l g}
\end{array}\right]^{(k)}=-\left[\begin{array}{c}
I_{j a} \\
I_{j b} \\
I_{j c} \\
I_{j g}
\end{array}\right]^{(k)}+\sum_{m \in M}\left[\begin{array}{l}
J_{m a} \\
J_{m b} \\
J_{m c} \\
J_{m g}
\end{array}\right]^{(k)}
$$

Where

$J_{l a}, J_{l b}, J_{l c}, J_{l g} \quad$ are current flows on line section $l$; and

3. Forward Sweep - nodal voltage calculation

Starting from the first layer and moving towards the last layer, the voltage at node $j$ is: 


$$
\left[\begin{array}{c}
V_{j a} \\
V_{j b} \\
V_{j c} \\
V_{j g}
\end{array}\right]^{(k)}=\left[\begin{array}{l}
V_{i a} \\
V_{i b} \\
V_{i c} \\
V_{i g}
\end{array}\right]^{(k)}-\left[\begin{array}{llll}
Z_{a a} & Z_{a b} & Z_{a c} & Z_{a g} \\
Z_{a b} & Z_{b b} & Z_{b c} & Z_{b g} \\
Z_{a c} & Z_{b c} & Z_{c c} & Z_{c g} \\
Z_{a g} & Z_{b g} & Z_{c g} & Z_{g g}
\end{array}\right]\left[\begin{array}{l}
J_{l a} \\
J_{l b} \\
J_{l c} \\
J_{l g}
\end{array}\right]^{(k)}
$$

\section{Convergence Test}

After steps 1,2 and 3 are executed in one iteration, the power mismatches at each node for all phases and ground are calculated:

$$
\begin{aligned}
& \Delta S_{i a}^{(k)}=V_{i a}^{(k)}\left(I_{i a}^{(k)}\right)^{*}-Y_{i a}^{*}\left|V_{i a}^{(k)}\right|^{2}-S_{i a} \\
& \Delta S_{i b}^{(k)}=V_{i b}^{(k)}\left(I_{i b}^{(k)}\right)^{*}-Y_{i b}^{*}\left|V_{i b}^{(k)}\right|^{2}-S_{i b} \\
& \Delta S_{i c}^{(k)}=V_{i c}^{(k)}\left(I_{i c}^{(k)}\right)^{*}-Y_{i c}^{*}\left|V_{i c}^{(k)}\right|^{2}-S_{i c} \\
& \Delta S_{i g}^{(k)}=V_{i g}^{(k)}\left(I_{i g}^{(k)}\right)^{*}
\end{aligned}
$$

If the real or imaginary part of any of the power mismatches is greater than a convergence criterion, steps 1, 2 and 3 are repeated until convergence is achieved.

\section{Flat Start}

The initial voltage for all nodes should be equal to the root node voltage:

$$
\left[\begin{array}{c}
V_{i a} \\
V_{i b} \\
V_{i c}^{(0)} \\
V_{i q}
\end{array}\right]^{2}=\left[\begin{array}{c}
V_{\text {ref }} \\
a^{2} \cdot V_{\text {ref }} \\
a \cdot V_{\text {ref }} \\
0
\end{array}\right] \quad, \quad a=e^{j \frac{2 \pi}{3}}
$$

where $V_{\text {ref }}$ is the reference voltage.

In case of single-wire DNER, the second and third row and column in the matrix equations (10)-(14) do not exist.

\section{Applications}

\section{a) Test network}

Test network used in case studies is the modified IEEE 34 bus radial DN [9,10], Fig. 4. Simplifying, the autotransformer $24.9 / 4.16 \mathrm{kV} / \mathrm{kV}$ in the original IEEE 34-bus test feeder is replaced with the line and the network is modeled with the single voltage level. The automatic 
voltage regulator is also not represented. General data are: frequency $f=60 \mathrm{~Hz}$, ground resistivity $\rho=100 \Omega-\mathrm{m}$. Network data for the single-wire case include: single-wire impedance $\bar{Z}_{a a}=(0.3679+j 0.6719) \Omega / \mathrm{km}$, impedance of the fictitious ground return "conductor" $\bar{Z}_{g g}=$ $(0.0592+j 0.4371) \Omega / \mathrm{km}$, mutual impedance between single wire and ground $\bar{Z}_{a g}=j 0.0740$ $\Omega / \mathrm{km}$. Network data for the three-wire case was obtained by the impedances calculation of the IEEE-34 original data [11], [12]. Both networks use a base voltage $V_{b}=24.9 \mathrm{kV}$, and reference voltage in the root node $V_{\text {ref }}=25.647 \mathrm{kV}$. Non-zero active $\left(\mathrm{P}_{\mathrm{L}}\right)$ and reactive $\left(\mathrm{Q}_{\mathrm{L}}\right)$ load and capacitors injection (Qc) are shown in Table I, Table II, Table III and Table IV for the single-wire and three-wire DNER, respectively.

\section{b) Case studies}

Several case studies have been performed using the extended power flow method in the considered IEEE test feeder. In this paper, power flow analysis in the single-wire and threewire DNER is presented. Two types of load models are applied: constant admittance and constant power.

Single-wire Case

By setting admittance $Y_{a}$ to be zero in considered single-wire DNER, we simulate Carson's model of the line (Fig.1).

\section{Three-wire Case}

In this case the extended power flow in $4 \times 4$ network representation is applied on the three-wire DNER using IEEE 34-node test feeder with unbalanced load. The main difference in operation single-wire and three-wire DNERs is the current through the ground. In case of single-wire DNER the current through the ground represents the opposite section current through the wire. However, in case of three-wire DNER, current through the ground is actually a current of the three-phase load unbalance. In case of three-wire DNER impact of the ground on the power flow solutions via calculation of the corrected impedance matrix, is investigated. Phase voltages obtained by three-phase power flow method from [7] and by the 
extended powe flow in $4 \times 4$ network representation, are compared. The influence of ground resistivity on the ground voltage and current is also investigated.

FIGURE 4.

TABLE 1.

TABLE 2.

TABLE 3.

TABLE 4.

\section{c) Results}

Phase and ground voltages and currents obtained by performing the extended power flow method on single-wire DNER are shown on Fig. 5 to Fig. 8.

Results obtained by performing the extended power flow method on three-wire DNER, are shown on Fig. 9 to Fig. 15. Differences in phase voltages obtained by ordinary three-phase power flow method [7] and the extended method are up to $1.5 \%$ (Fig. 9, Fig. 12). Fig. 13 and Fig. 14 show ground voltages for different ground resistivity $(50-5000 \Omega-m)$, and constant power and constant admittance load modelling, respectively. Fig. 15 shows ground current in three-wire DNER for constant power and constant admittance load modelling.

Generally, load modeling plays significant role in the power flow simulations. The results show a large deference in phase voltages due to load model and minor deference due to the line model.

Considering three-wire DNER, constant admittance load model gives higher voltages and currents than the constant power load model. Shunt admittances of phase lines $Y_{a}, Y_{b}, Y_{c}$ affect the power flow solution in sense of increasing ground voltages and ground currents.

Used backward-forward technique has not lost its convergence speed, although the computation time increased. There is the speed penalty to be paid for using $4 \times 4$ network representation. 
FIGURE 5.

FIGURE 6.

FIGURE 7.

FIGURE 8.

FIGURE 9.

FIGURE 10.

FIGURE 11.

FIGURE 12.

FIGURE 13.

FIGURE 14.

FIGURE 15.

\section{Conclusions}

In this paper, a power flow algorithm in the single-wire and three-wire DNER, based on backward-forward technique [7], is presented. In this novel use of the technique the ground is explicitly represented. Besides, an iterative method for determining impedance for modelling ground in the DNER, based on voltage and current measurement in the simple test system and proposed power flow analysis, is suggested. The extended power flow technique, based on the $2 \times 2$ representation in the single phase DNERs or $4 \times 4$ representation in the three phase DNERs, enables the user to investigate the effects of ground on the operation of the distribution systems with earth return.

The software package based on the extended power flow technique can be an efficient tool for power quality studies, safety analysis, short circuit analysis and other applications in DNs with earth return, where ground current and voltages are of particular interest, mainly.

\section{ACKNOWLEDGMENT}

The authors would like to thank UNESP, Faculty of Engineering of Ilha Solteira, SP, Brazil, for the provision of facilities. R. M. Ciric is grateful to the Foundation of Assistance to the Research of the State of Sao Paulo (FAPESP) for its financial support to undertake this 
research (Project no. 00/12099-1). The authors also would like to thank the ELUCID Solutions S.A., Sao Paulo, SP, Brazil, for their financial support and commitment to this project (FEPISA 003-2001).

\section{APPENDIX}

\section{DETERMINING GROUND IMPEDANCE}

The proposition of using described power flow for DNER is that self ground impedance $\bar{Z}_{g g}$ and mutual ground impedance $\bar{Z}_{a g}$ are known. Experience from the operation of the DNER shows that ground resistivity $\rho$ is not only varying function of soil type and humidity, but a daily varying function of time, as well. Consequently in practice, the impedance $\bar{Z}_{a g}$ in the DNERs is not constant, but time varying function, as well. The question is: How to determine impedance $\bar{Z}_{g g}$ and $\bar{Z}_{a g}$ of the considered DNER needed in the proposed power flow algorithm? Fig. A.1 shows the suggested iterative method for determining impedance $\bar{z}_{g g}$ and $\bar{z}_{a g}$ of the DNER. The suggested iterative method is based on voltage and current measurement in the real-life two-bus test network from Fig. 2 and exhaustive power flow calculation. The measurement set consists of the voltages and currents in the feeder head and on the consumer side of the network: $V_{a}, I_{a}, V_{a}$ and $I_{g}$. In the proposed method, Carson's equations are used to calculate the first iteration of the impedance $\bar{Z}_{g g}$ and $\bar{Z}_{a g}$. Ground resistivity $\rho$ for the considered type of soil is used as the initial value. Then, power flow in the considered real-life two-bus single-wire DNER from Fig. 2 is performed and the obtained data and the data from the measurements are compared. Correction of the $\bar{Z}_{g g}$ and $\bar{Z}_{a g}$ is performed using the voltage and current mismatches on the consumer side. The procedure is repeated until the satisfied accordance between calculated and measured voltage and current values on the customer side is achieved. Finally, more precise ground resistivity, as a daily 
function of time $\rho(t)$, can be obtained using the calculated mutual ground impedance $\bar{Z}_{a g}$. The method for determining the ground impedance still needs an extensive field tests.

\section{FIGURE A.1}

\section{REFERENCES}

[1] Carson JR. Wave propagation in overhead wires with ground return. Bell System Technical Journal 1926.

[2] Carson JR. Wave propagation in overhead wires with ground return. Bell System Technical Journal 1926. In the Proceedings of the Thrity-Second Annual North American Power Symposium. Waterloo, Canada, 2000. p. P-65-P-80.

[3] Kersting WH. Distribution System Modeling and Analysis. CRC Press 2002.

[4] Anderson PM. Analysis of Faulted Power Systems. New York: IEEE Press Power Systems Engineering Series, 1995. p. 71-83.

[5] Lakervi E, Holmes EJ. Electricity Distribution Network Design. IEE Power Engineering Series 21. London: Peter Peregrines Ltd. On behalf of the IEE, 1995.

[6] Shirmohammadi D, Hong HW, Semlyen A, Luo GX. A compensation-based power flow method for weakly meshed distribution and transmission networks. IEEE Trans. on Power Systems 1988; 3(2):753-762.

[7] Cheng CS, Shirmohammadi D. A three-phase power flow method for real-time distribution system analysis. IEEE Trans. on Power Systems 1995; 10(2):671-769.

[8] Luo GX, Semlyen A. Efficient load flow for large weakly meshed networks. IEEE Trans. on Power Systems 1990; 5(4):1309-1316.

[9] Rajicic D, Ackovski R, Taleski R. Voltage correction power flow. IEEE Trans. on Power Delivery 1994; 9(2):1056-1062. 
[10]Rajicic D, Dimitrovski A. A new method for handling PV nodes in backward/forward power flow for radial and weakly meshed networks. IEEE Porto Power Tech Conference 2001, paper PS03-286.

[11]IEEE Distribution Planning Working Group Report. Radial distribution test feeders. IEEE Trans. on Power Systems 1991; 6(3):975-985.

[12] IEEE Distribution System Analysis Subcommittee Report. Radial distribution test feeders. PES Summer Meeting 2000.

Available: http://ewh.ieee.org/soc/pes/dsacom/testfeeders.html 


\section{Figure Captions}

Fig. 1. Carson's line

Fig. 2. Model of the single-wire earth return distribution line

Fig. 3. Model of the three-wire earth return distribution line

Fig. 4. IEEE 34-node test feeder

Fig. 5. Phase to Ground Voltages in single-wire DNER using Constant Power (CP) and Constant Admittance (CA) Load Modelling.

Fig. 6. Ground Voltages in single-wire DNER using Constant Power (CP) and Constant Admittance (CA) Load Modelling.

Fig. 7. Ground Currents in single-wire DNER using Constant Power (CP) and Constant Admittance (CA) Load Modelling.

Fig. 8. Ground Voltages in single-wire DNER for different ground resistivity and Constant Admittance Load Modelling.

Fig. 9. Phase Voltages in three-wire DNER using the extended power flow method: Constant Power Load Modelling.

Fig. 10. Phase Voltages in three-wire DNER using extended power flow method: Constant Admittance Load Modelling.

Fig. 11. Phase Voltages in the three-wire DNER using ordinary three-phase power flow method: Constant Admittance Load Modelling.

Fig. 12. Phase Voltages in three-wire DNER using ordinary three-phase power flow method: Constant Power Load Modelling.

Fig. 13. Ground Voltages in three-wire DNER using different ground resistivity and Constant Power Load Modelling.

Fig. 14. Ground Voltages in three-wire DNER using different ground resistivity and Constant Admittance Load Modelling.

Fig. 15. Ground Current in three-wire DNER using Constant Power (CP) and Constant Admittance (CA) Load Modelling.

Fig. A.1. Suggested method for determining ground impedance in DNERs 


\section{Table Captions}

TABLE I. Active and Reactive Injections in the considered single-wire DNER (non-zero elements)

TABLE II. Capacitors Injections in the considered single-wire DNER (non-zero elements)

TABLE III. Active and Reactive Injections in the considered three-wire DNER (non-zero elements)

TABLE IV. Capacitors Injections in the considered three-wire DNER (non-zero elements) 


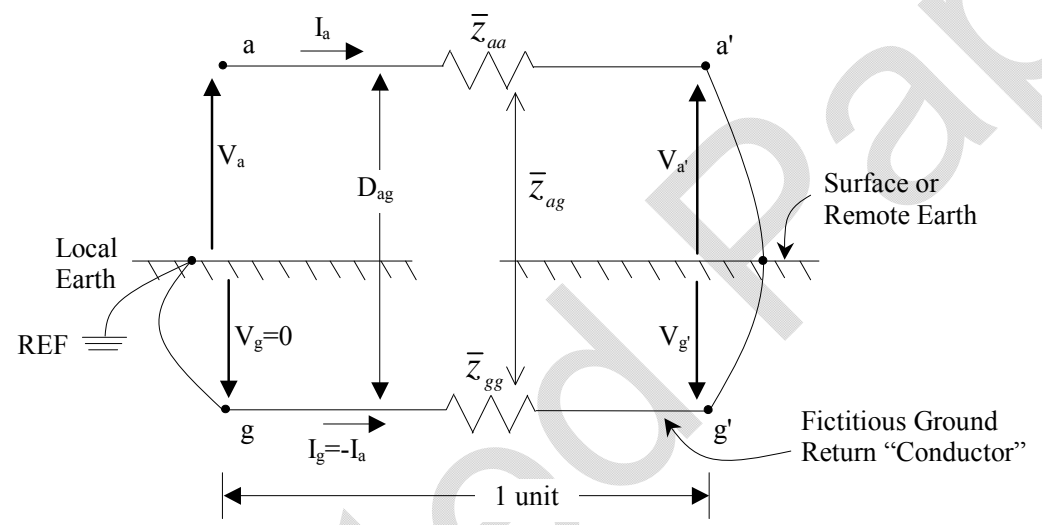

Fig. 1. Carson's line 


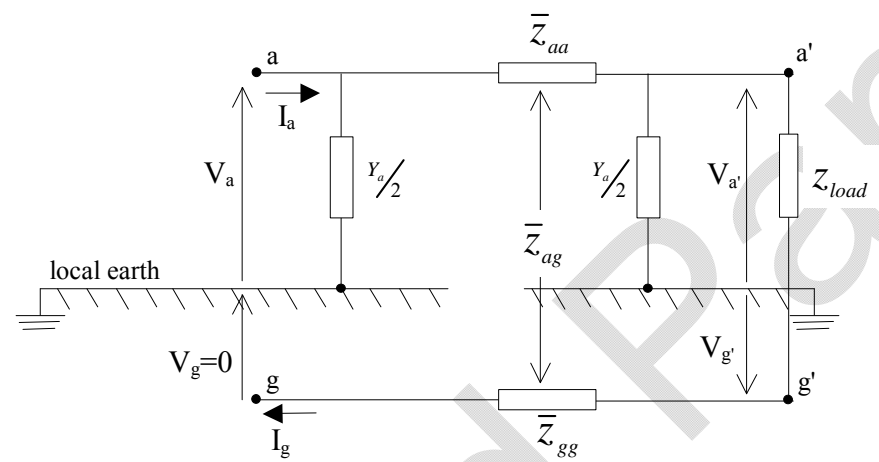

Fig. 2. Model of the single-wire earth return distribution line 


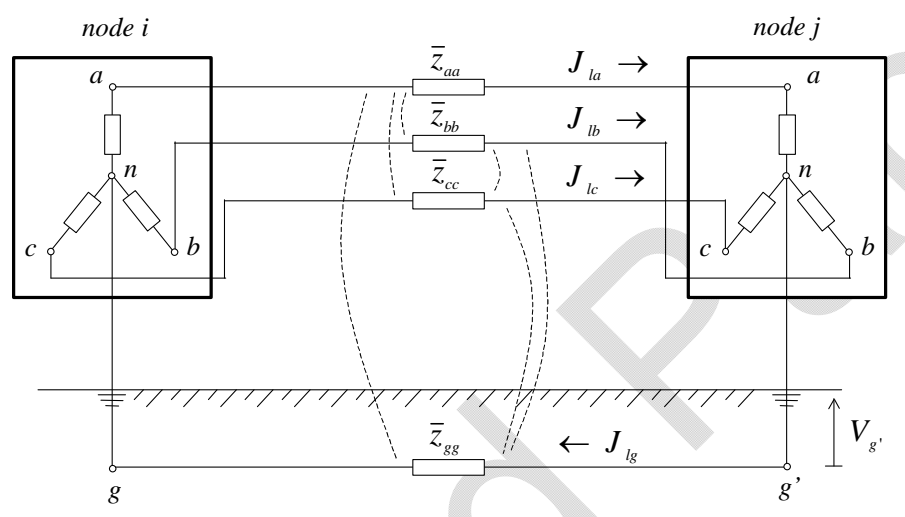

Fig. 3. Model of the three-wire earth return distribution line 


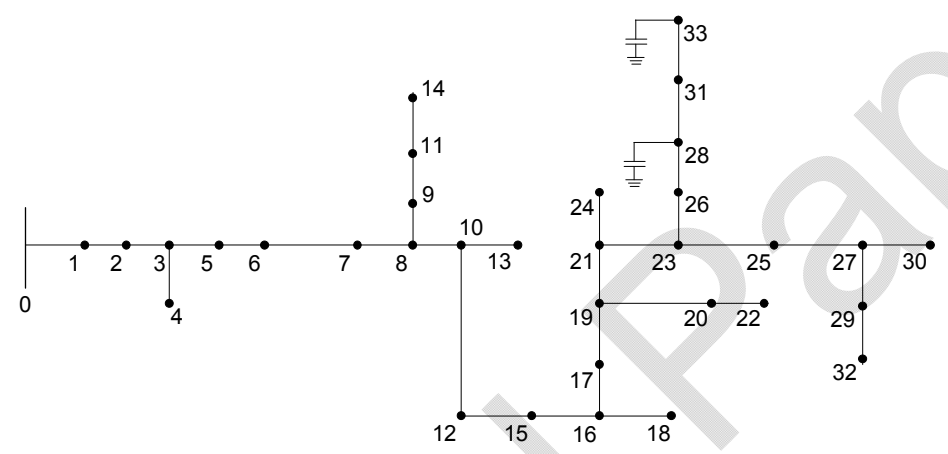

Fig.4. IEEE 34-node test feeder 
TABLE I

ACTIVE AND REACTIVE INJECTIONS IN THE CONSIDERED SINGLE-WIRE DNER (NON-ZERO ELEMENTS)

\begin{tabular}{|c|r|r|}
\hline node & $\mathrm{P}_{\mathrm{L}}(\mathrm{kW})$ & $\mathrm{Q}_{\mathrm{L}}(\mathrm{kVAr})$ \\
\hline 9 & 17.0 & 8.5 \\
\hline 11 & 84.5 & 43.5 \\
\hline 12 & 3.5 & 1.5 \\
\hline 14 & 67.5 & 35.0 \\
\hline 15 & 13.5 & 6.5 \\
\hline 19 & 3.5 & 1.5 \\
\hline 21 & 6.5 & 3.0 \\
\hline 22 & 150.0 & 75.0 \\
\hline 23 & 10.0 & 5.0 \\
\hline 24 & 1.0 & 0.5 \\
\hline 25 & 43.0 & 27.5 \\
\hline 26 & 4.5 & 2.5 \\
\hline 27 & 24.0 & 12.0 \\
\hline 28 & 139.5 & 107.5 \\
\hline 30 & 18.0 & 11.5 \\
\hline 33 & 20.0 & 16.0 \\
\hline
\end{tabular}


TABLE II

CAPACITORS INJECTIONS IN THE CONSIDERED SINGLE-WIRE DNER (NON-ZERO ELEMENTS)

\begin{tabular}{|c|c|}
\hline node & $\mathrm{Q}_{\mathrm{C}}(\mathrm{kVAr})$ \\
\hline 28 & 100.0 \\
\hline
\end{tabular}

\begin{tabular}{|l|l|}
\hline 28 & 150.0 \\
\hline 33 & 150.0 \\
\hline
\end{tabular}


TABLE III

ACTIVE AND REACTIVE INJECTIONS IN THE CONSIDERED THREE-WIRE DNER (NON-ZERO ELEMENTS)

\begin{tabular}{|c|r|r|r|r|r|r|}
\hline node & $P_{\text {La }}(\mathrm{kW})$ & $Q_{\text {La }}(\mathrm{kVAr})$ & $P_{\mathrm{Lb}}(\mathrm{kW})$ & $\mathrm{Q}_{\mathrm{Lb}}(\mathrm{kVAr})$ & $\mathrm{P}_{\mathrm{Lc}}(\mathrm{kW})$ & $\mathrm{Q}_{\mathrm{Lc}}(\mathrm{kVAr})$ \\
\hline 1 & 0.0 & 0.0 & 15.0 & 7.5 & 12.5 & 7.0 \\
\hline 2 & 0.0 & 0.0 & 15.0 & 7.5 & 12.5 & 7.0 \\
\hline 3 & 0.0 & 0.0 & 8.0 & 4.0 & 0.0 & 0.0 \\
\hline 4 & 0.0 & 0.0 & 8.0 & 4.0 & 0.0 & 0.0 \\
\hline 8 & 0.0 & 0.0 & 2.5 & 1.0 & 0.0 & 0.0 \\
\hline 9 & 17.0 & 8.5 & 0.0 & 0.0 & 0.0 & 0.0 \\
\hline 10 & 0.0 & 0.0 & 22.5 & 11.0 & 2.0 & 1.0 \\
\hline 11 & 84.5 & 43.5 & 0.0 & 0.0 & 0.0 & 0.0 \\
\hline 12 & 3.5 & 1.5 & 0.0 & 0.0 & 2.0 & 1.0 \\
\hline 13 & 0.0 & 0.0 & 20.0 & 10.0 & 0.0 & 0.0 \\
\hline 14 & 67.5 & 35.0 & 0.0 & 0.0 & 0.0 & 0.0 \\
\hline 15 & 13.5 & 6.5 & 12.0 & 6.0 & 25.0 & 10.0 \\
\hline 16 & 0.0 & 0.0 & 2.0 & 1.0 & 0.0 & 0.0 \\
\hline 19 & 3.5 & 1.5 & 1.0 & 0.5 & 3.0 & 1.5 \\
\hline 21 & 6.5 & 3.0 & 8.5 & 4.5 & 9.5 & 5.0 \\
\hline 22 & 150.0 & 75.0 & 150.0 & 75.0 & 150.0 & 75.0 \\
\hline 23 & 10.0 & 5.0 & 17.5 & 9.0 & 61.5 & 31.0 \\
\hline 24 & 1.0 & 0.5 & 0.0 & 0.0 & 0.0 & 0.0 \\
\hline 25 & 43.0 & 27.5 & 35.0 & 24.0 & 96.0 & 54.5 \\
\hline 26 & 4.5 & 2.5 & 0.0 & 0.0 & 0.0 & 0.0 \\
\hline 27 & 24.0 & 12.0 & 16.0 & 8.5 & 21.0 & 11.0 \\
\hline 28 & 139.5 & 107.5 & 147.5 & 111.0 & 145.0 & 110.5 \\
\hline 29 & 0.0 & 0.0 & 14.0 & 7.0 & 0.0 & 0.0 \\
\hline 30 & 18.0 & 11.5 & 20.0 & 12.5 & 9.0 & 7.0 \\
\hline 31 & 0.0 & 0.0 & 24.0 & 11.5 & 0.0 & 0.0 \\
\hline 32 & 0.0 & 0.0 & 14.0 & 7.0 & 0.0 & 0.0 \\
\hline 33 & 20.0 & 16.0 & 31.5 & 21.5 & 20.0 & 16.0 \\
\hline
\end{tabular}


TABLE IV

CAPACITORS INJECTIONS IN THE CONSIDERED THREE-WIRE DNER (NON-ZERO ELEMENTS)

\begin{tabular}{|c|c|c|c|}
\hline node & $\mathrm{Q}_{\mathrm{Ca}}(\mathrm{kVAr})$ & $\mathrm{Q}_{\mathrm{Cb}}(\mathrm{kVAr})$ & $\mathrm{Q}_{\mathrm{Cc}}(\mathrm{kVAr})$ \\
\hline 28 & 100.0 & 100.0 & 100.0 \\
\hline 33 & 150.0 & 150.0 & 150.0 \\
\hline
\end{tabular}




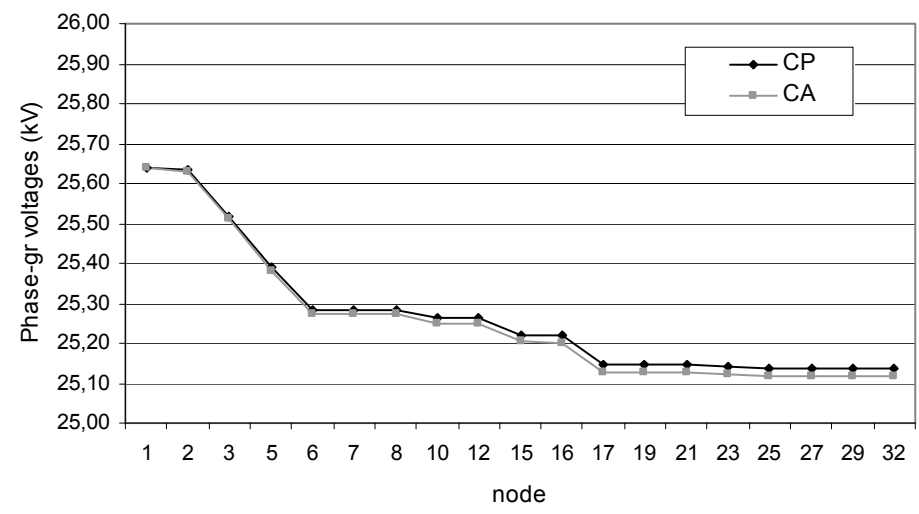

Fig. 5. Phase to Ground Voltages in single-wire DNER using Constant Power (CP) and Constant Admittance (CA) Load Modelling. 


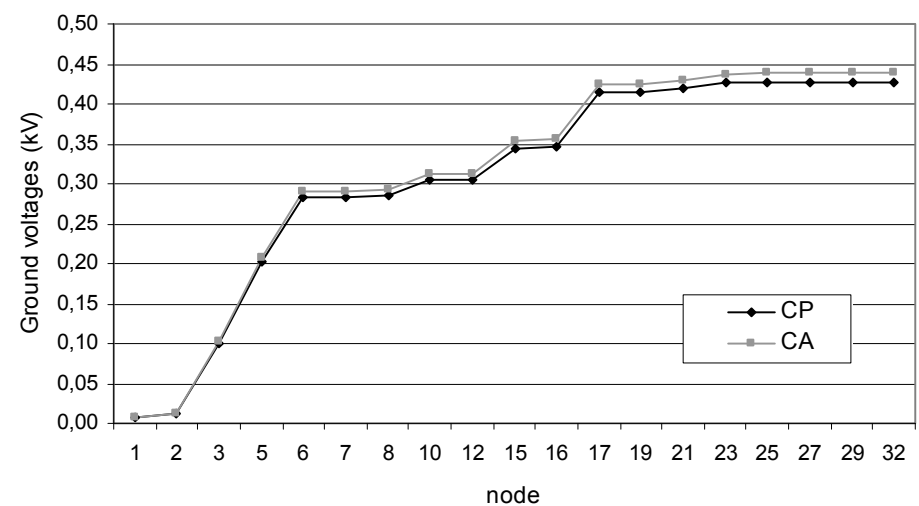

Fig. 6. Ground Voltages in single-wire DNER using Constant Power (CP) and Constant Admittance (CA) Load Modelling. 


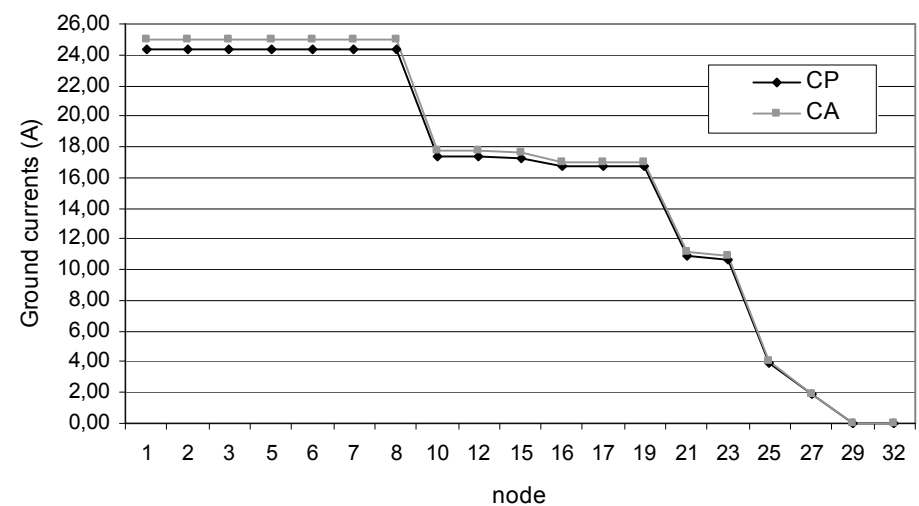

Fig. 7. Ground Currents in single-wire DNER using Constant Power (CP) and Constant Admittance (CA) Load Modelling. 


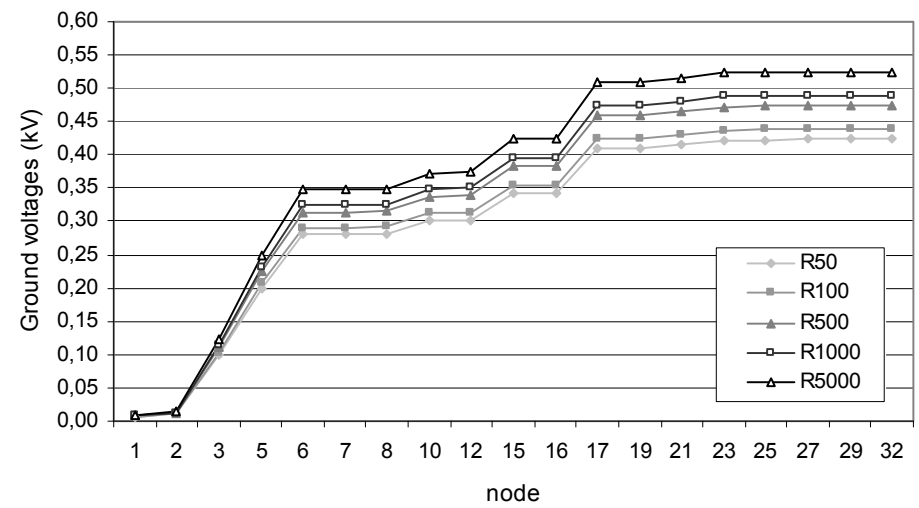

Fig. 8. Ground Voltages in single-wire DNER for different ground resistivity and Constant Admittance Load Modelling. 


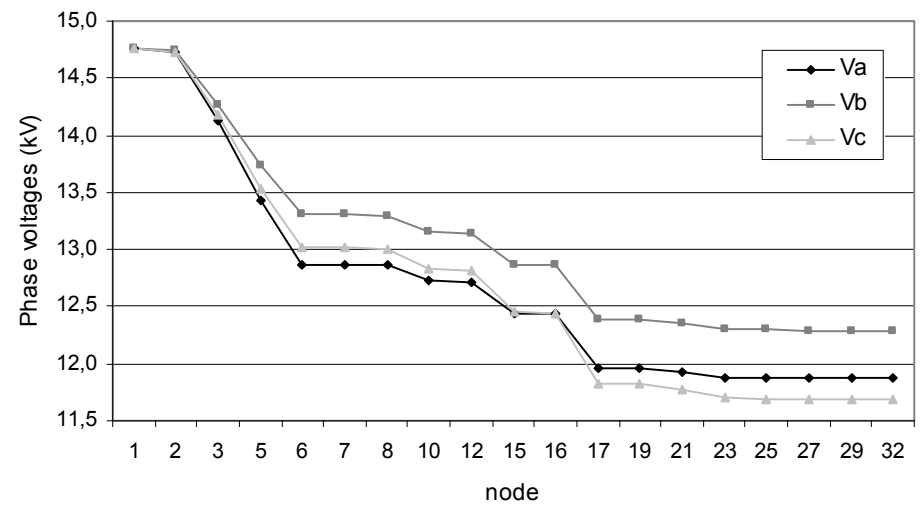

Fig. 9. Phase Voltages in three-wire DNER using the extended power flow method: Constant Power Load Modelling. 


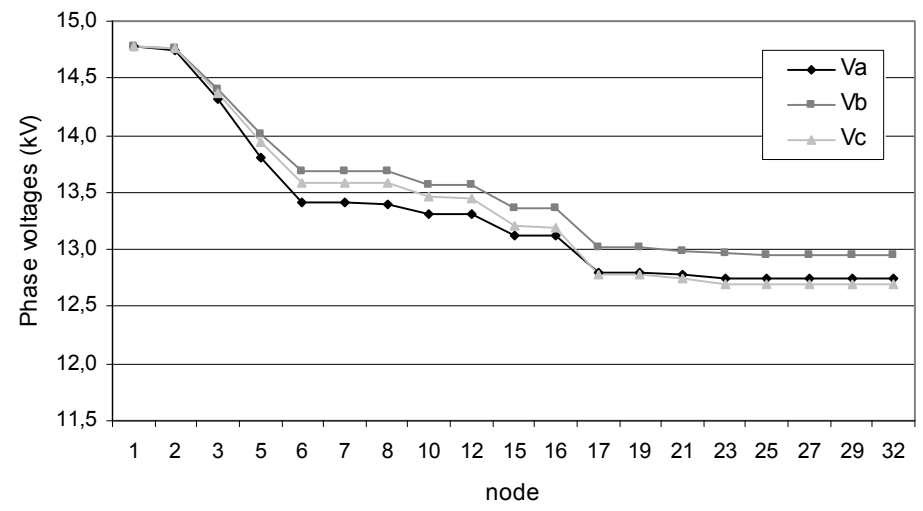

Fig. 10. Phase Voltages in three-wire DNER using extended power flow method: Constant Admittance Load Modelling. 


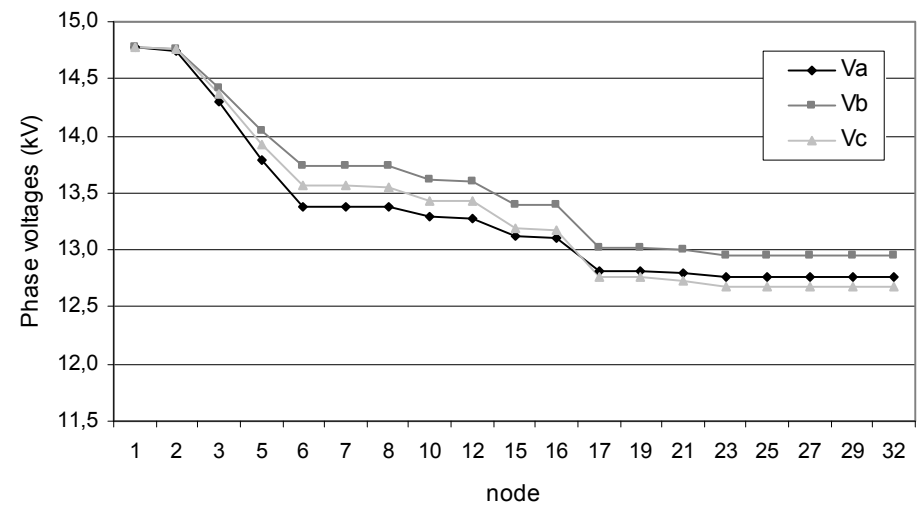

Fig. 11. Phase Voltages in the three-wire DNER using ordinary three-phase power flow method: Constant Admittance Load Modelling. 


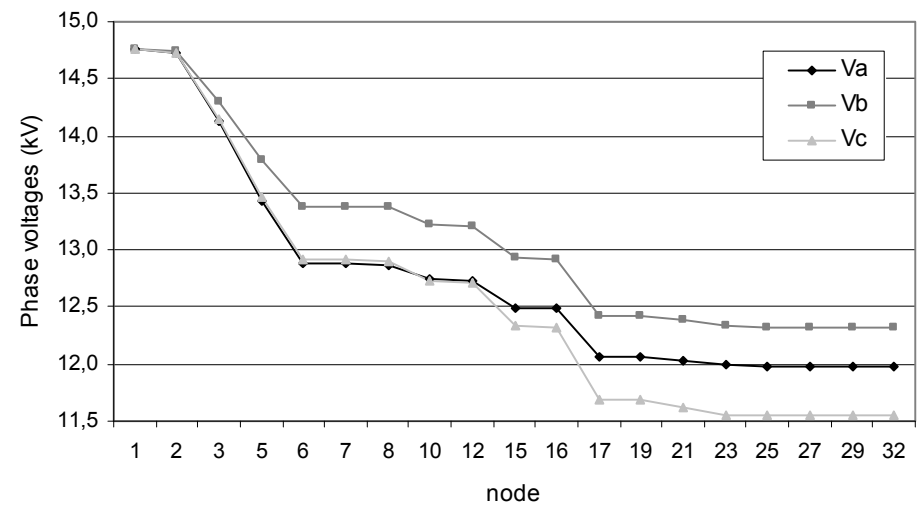

Fig. 12. Phase Voltages in three-wire DNER using ordinary three-phase power flow method: Constant Power Load Modelling. 


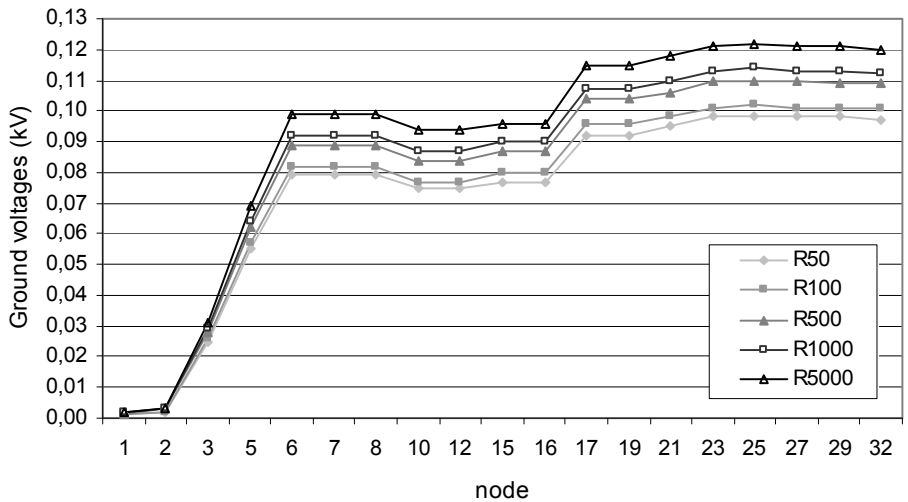

Fig. 13. Ground Voltages in three-wire DNER using different ground resistivity and Constant Power Load Modelling. 


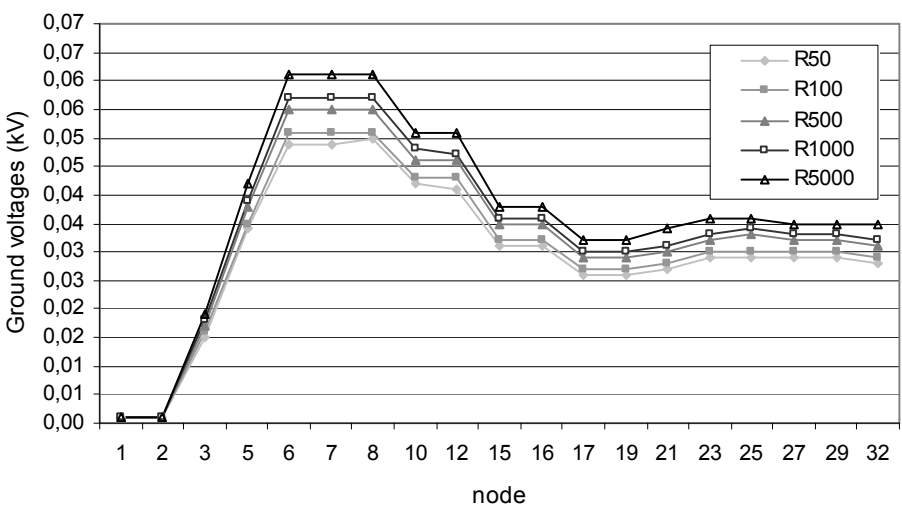

Fig. 14. Ground Voltages in three-wire DNER using different ground resistivity and Constant Admittance Load Modelling. 


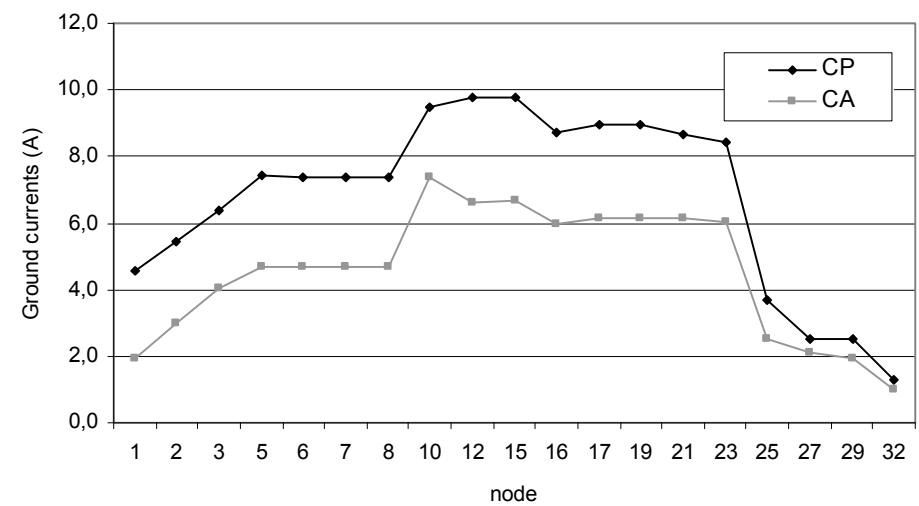

Fig. 15. Ground Current in three-wire DNER using Constant Power (CP) and Constant Admittance (CA) Load Modelling. 


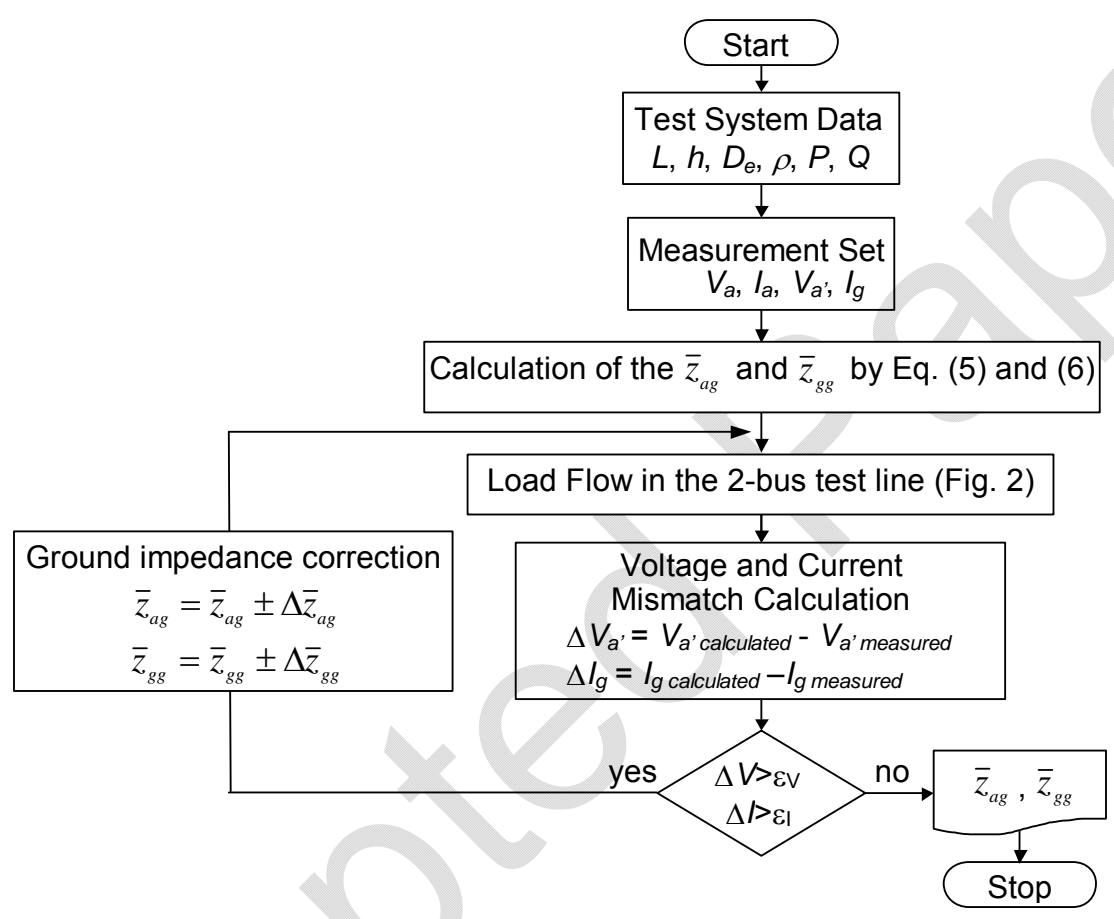

Fig. A.1. Suggested method for determining ground impedance in DNERs 